\title{
Imaging interhemispheric interactions in normal and monocularly deprived young visual cortex by manganese-enhanced magnetic resonance imaging
}

\author{
Shu-Juan Fan ${ }^{1,2}$, Alex T.L. Leong ${ }^{1,2}, \mathrm{Ed} \mathrm{X} \mathrm{Wu}^{1,2 *}$
}

\begin{abstract}
This study employed in vivo high resolution manganese-enhanced magnetic resonance imaging (MEMRI) to investigate interhemispheric interactions in normal and monocularly deprived visual cortex of rats with unimpaired vision, left or right eyelid suturing, or left or right eye enucleation. After seven days with altered vision, the animals were injected with manganese ions into the right visual cortex and imaged with an inversion-recovery modified driven-equilibrium Fourier transform sequence. The results showed prominent manganese transport, via the splenium of the corpus callosum, to the left hemisphere where the signal enhancement peaked on the border of the primary/secondary visual cortex and expanded medial-laterally with layer specificity. Significant decrease of transcallosal manganese labeling of the left hemisphere was observed after left eyelid suturing, left eye enucleation, and right eyelid suturing but not right eye enucleation, suggesting that the adult brain recruits different mechanisms for adaptation under different circumstances. Our findings demonstrated MEMRI as an efficient tool for investigating interhemispheric interactions both anatomically and functionally.
\end{abstract}

Keywords: interhemispheric interactions, manganese-enhanced MRI, visual cortex, monocular eyelid suturing, monocular enucleation, albino rats

\section{INTRODUCTION}

Understanding cortical interhemispheric interactions and plasticity in the adult brain is important because it can provide a rational basis for therapies against diseases such as amblyopia $(1,2)$, cognitive dysfunction in Down's syndrome (3), and functional visuospatial neglect caused by unilateral brain damage (4). Visual cortex is perhaps the most intensively studied and best understood neocortical area. Unlike other cortical areas, neuronal responses of the visual cortex are strictly different from its subcortical inputs (5). Such uniqueness enables researchers to distinguish cortical and subcortical changes and pursue fundamental questions regarding cortical development and plasticity under normal and altered conditions. The corpus callosum is the main white matter structure in the mammalian brain for connecting cortical areas of the two hemispheres. Specifically in visual cortex, callosal cells are densely located in the transition zone of the primary/secondary visual cortex (V1/V2 transition zone) (6), and project axons to an even narrower area in the contralateral hemisphere (7). These callosal connections play a pivotal role in interhemispheric interactions that are essential for maintaining the anatomical and functional continuity between the split representations of the visual fields (7), and contribute to binocularity and mediate ocular dominance plasticity (8). Binocularity is one of the most prominent characteristics of visual cortical neurons which are capable of responding to only one or both eyes (9). Ocular dominance plasticity is a phenomenon where binocularly responsive cortical neurons become abnormally more responsive to one eye over the other. Such plasticity has been widely observed and extensively studied in young primates $(10)$, kittens $(11)$, rabbits $(12)$, and rodents $(8,13)$ during early development. Recently, it has also been observed in adult rodent brains upon prolonged manipulations, however, with heterogeneous findings regarding layer specificity $(14,15)$ and deprived eye response changes $(16,17)$. Mixed findings also exist regarding the cellular basis of interhemispheric interactions via callosal axons $(18,19)$. These discrepancies likely arose from

\footnotetext{
${ }^{1}$ Laboratory of Biomedical Imaging and Signal Processing, The University of Hong Kong, Hong Kong SAR, China. ${ }^{2}$ Department of Electrical and Electronic Engineering, The University of Hong Kong, Hong Kong SAR, China.

*Corresponding author. Email: ewu@eee.hku.hk (E.X.W.)
}

the intrinsic technical limitations encountered in these electrophysiological or histological studies, namely, finite area and depth of cortical sampling, use of different anesthesia regimens, and targeting of limited cortical circuit components.

Manganese-enhanced magnetic resonance imaging (MEMRI), by utilizing paramagnetic manganese $\left(\mathrm{Mn}^{2+}\right)$ ion as a calcium analog that can enter excitable cells (20-22), has been increasingly applied for detecting and measuring neuronal activity (23-26), plasticity (27-29), and neurodegeneration (30). It has also been employed extensively for axonal tract tracing (21,31-34). Recently, MEMRI has been demonstrated to be capable of tracing layerspecific interhemispheric callosal connections in the somatosensory cortex (35), and sensitive enough to detect subtle layer-specific activity changes in the visual cortex during light stimulation upon systemic $\mathrm{Mn}^{2+}$ administration (36). Thus MEMRI potentially allows in vivo tracing of normal and altered interhemispheric interactions in the visual cortex following experiential manipulations.

Natural stimuli, voluntary motor activities, and electrical stimulation are known to induce and increase $\mathrm{Mn}^{2+}$ uptake, transport and accumulation in motor, olfactory, and vocal control systems in MEMRI $(22,37,38)$. However, retinal blindness was observed not to affect $\mathrm{Mn}^{2+}$ uptake in the retina and its transport in the optic nerve/tract after intravitreal injection (39). This suggests that $\mathrm{Mn}^{2+}$ transport in different neuronal pathways may be modulated by activity to different extents. Alternatively, it implies that the sensitivity of MEMRI for tracing activity-modulated neuronal interactions is dependent of the route and dosage of $\mathrm{Mn}^{2+}$ administration (40). Therefore, it is imperative to investigate whether interhemispheric $\mathrm{Mn}^{2+}$ transport is sensitive to visual activity changes upon intracortical $\mathrm{Mn}^{2+}$ administration. Recently, Chan et al. have demonstrated interhemispheric $\mathrm{Mn}^{2+}$ transport in the visual cortex (41). By employing high-resolution 3D MEMRI using an inversion-recovery modified driven-equilibrium Fourier transform (MDEFT) sequence, the current study extended this preliminary study with three objectives: (a) to characterize the layer-specific interhemispheric $\mathrm{Mn}^{2+}$ transport in normal rats; (b) to determine whether interhemispheric $\mathrm{Mn}^{2+}$ transport is sensitive to activity modulation and can serve as a functional marker for interhemispheric interactions; and (c) to document how the 
interhemispheric interactions alter upon visual deprivation by eyelid suturing or eye enucleation in the dominant or non-dominant eye.

\section{RESULTS}

\section{Characteristics of $\mathrm{Mn}^{2+}$ enhancement in the left hemisphere} upon intracortical $\mathrm{Mn}^{2+}$ administration in the right visual cortex in normal adult rats

Before $\mathrm{MnCl}_{2}$ injection, the corpus callosum consistently showed lower signal intensity than the surrounding white matter (Fig. 1, left panel, arrowheads). At $8 \mathrm{hrs}$ after injection, it exhibited significant enhancement and was clearly demarcated. At $24 \mathrm{hrs}$ after injection and later, it gradually became less distinguished due to the increased enhancement of its surrounding white matter.

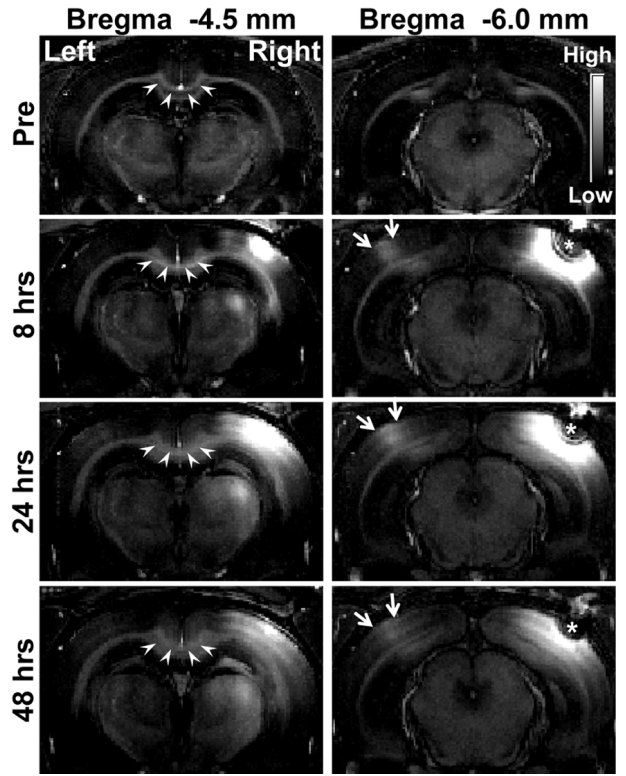

Fig. 1. Typical MDEFT images acquired at 24 hours before (Pre), 8 hours ( 8 hrs), 24 hours (24 hrs), and 48 hours (48 hrs) after $\mathrm{Mn}^{2+}$ injection. Corpus callosum (left panel, arrowheads) showed lower signal intensity than surrounding white matter before injection and robust enhancement at $8 \mathrm{hrs}$ after injection. Enhancement in the contralateral visual cortex appeared as a narrow stripe (right panel, enclosed by arrows), which widened at $24 \mathrm{hrs}$ and diminished in signal intensity at $48 \mathrm{hrs}$. Hypointensity at the injection core (right panel, white colored " ")was caused by severe local magnetic field distortion due to highly concentrated $\mathrm{Mn}^{2+}$

Meanwhile, the left hemisphere exhibited significant enhancement at all three time points after injection, distinctly at the location (Fig. 1, right panel, enclosed by arrows) that mirrored the injection site (Fig. 1, right panel, white colored “*”). Such enhancement showed a narrow stripe pattern at $8 \mathrm{hrs}$. It then expanded mediallaterally with increased signal intensity and exhibited a prominent bi-laminar pattern at $24 \mathrm{hrs}$. It further expanded with decreased signal intensity at $48 \mathrm{hrs}$.

Further analysis showed that the $\mathrm{Mn}^{2+}$ enhanced area in the left hemisphere corresponded to the V1/V2 transition zone (42), an area that is known to contain abundant callosal projections (Figs. 2A \& 2D, green lines) (35). The three-dimensionally reconstructed MDEFT images clearly depicted the $\mathrm{Mn}^{2+}$ transport pathway via corpus callosum at 8 hrs after injection (Fig. 2B, white arrowheads). It originated from the injected/right hemisphere as a thick band, first went rostrally, then caudally after crossing the midline (Fig. 2B, inset) and finally terminated abruptly at the V1/V2 border (Fig. 2B, red arrowhead). It remained discernible at $24 \mathrm{hrs}$ after injection (Fig. 2C). Temporally, the enhancement in the left V1/V2

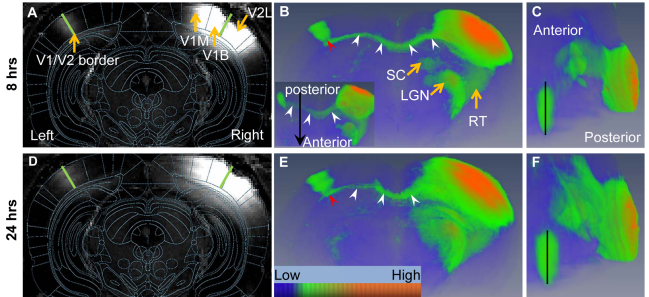

Fig. 2. MDEFT images overlaid on the standard rat brain atlas at bregma $-6.0 \mathrm{~mm}$ (A, D) and reconstructed 3D MDEFT color images showing the coronal view of the transcallosal $\mathrm{Mn}^{2+}$ transport pathway via the corpus callosum $(\mathrm{B}, \mathrm{E})$ and the spread of $\mathbf{M n}^{2+}$ enhancement (C, F). Green lines in panels A and D depict the V1/V2 border. Black lines in panels $C$ and $F$ show the medial-lateral spread of the contralateral hemisphere enhancement over time. The inset in panel $B$ illustrates the $\mathrm{Mn}^{2+}$ transport pathway viewing from the cranial base. All images were from one animal. Panels A-C and D-F correspond to the results at $8 \mathrm{hrs}$ and $24 \mathrm{hrs}$ after $\mathrm{Mn}^{2+}$ injection, respectively. LGN: lateral geniculate nucleus; SC: superior colliculus; $\mathrm{RT}$ : retinothalamic projections.

transition zone largely spread medial-laterally (Figs. 2B \& 2E, red arrowhead), not anterior-posteriorly (Figs. 2C \& 2 F) while the ipsilateral enhancement exhibited relatively isotropic expansion.

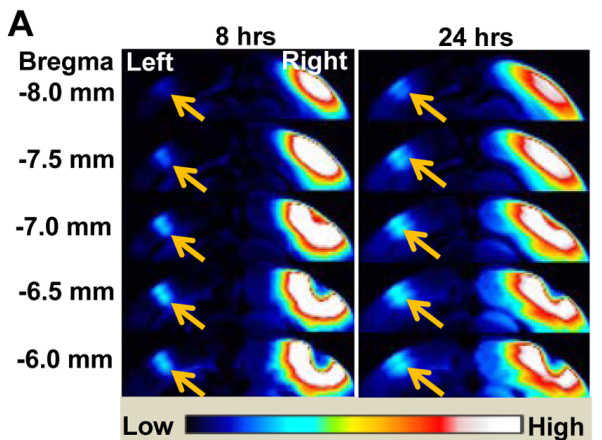

B
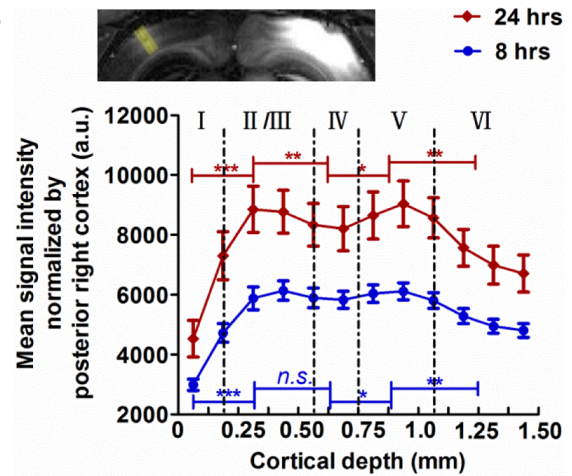

C

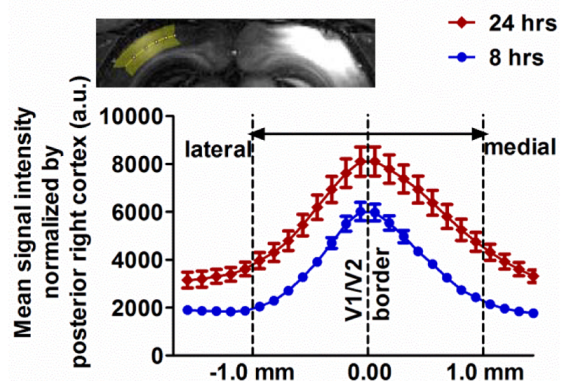

Fig. 3. Five consecutive color-coded $2 D$ MDEFT images from one normal rat. Prominent $\mathrm{Mn}^{2+}$ enhancement in the left hemisphere (A), and layer-specific $\mathrm{Mn}^{2+}$ enhancement along the cortical depth direction (B) and the medial-lateral direction (C) within left V1/V2 transition zone. Data are shown as mean \pm standard error of mean $(n=5)$. 
The stripe-like bi-laminar $\mathrm{Mn}^{2+}$ enhancement pattern in the left V1/V2 transition zone was analyzed semi-quantitatively in five normal controls at $8 \mathrm{hrs}$ and $24 \mathrm{hrs}$ after injection (Fig. 3) by plotting the pixel-by-pixel signal intensity in five consecutive slices (Fig. 3A) along the cortical depth direction (Fig. 3B) or mediallaterally along the cortical surface (Fig. 3C). The cortex was divided into six layers based on published cortical thickness values $(36,43)$. The enhancement peaked in layers II/III and V at both 8 and 24 hrs, but exhibited the best layer IV demarcation at $24 \mathrm{hrs}$. Mediallaterally, the enhancement symmetrically decreased from the V1/V2 border and was confined within approximately $1 \mathrm{~mm}$ from the V1/V2 border.

Decreases of $\mathrm{Mn}^{2+}$ enhancement in the left V1/V2 transition zone upon left/right eyelid suturing and left eye enucleation

Both left and right eyelid suturing (LS and RS respectively) significantly reduced $\mathrm{Mn}^{2+}$ enhancement in the left V1/V2 transition zone at 24 hrs (Figs. 4A \& 4B).

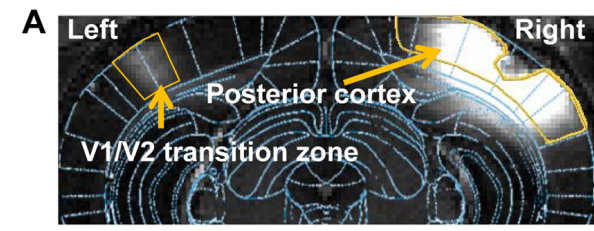

B

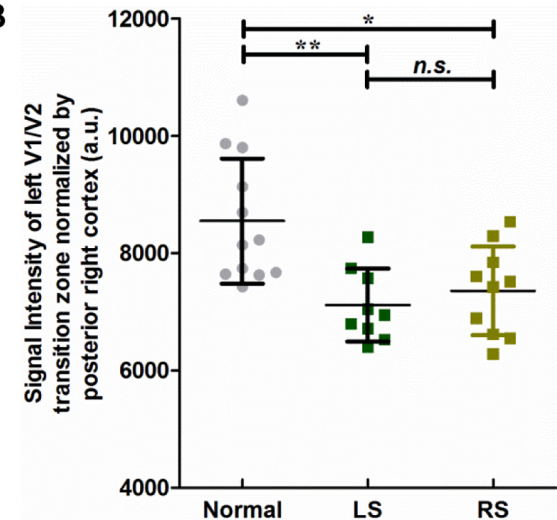

C

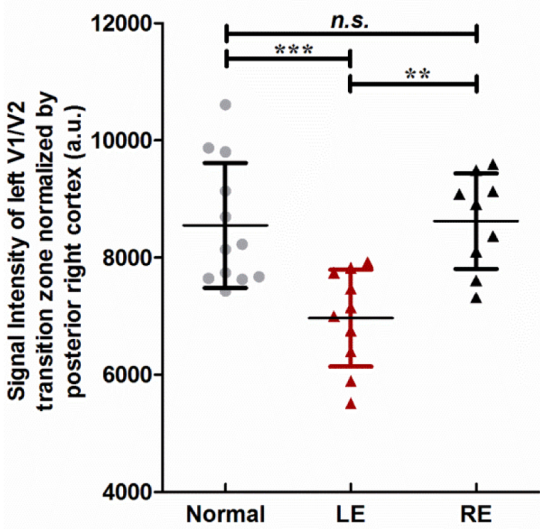

Fig. 4. (A) MDEFT images acquired at $24 \mathrm{hrs}$ after $\mathrm{Mn}^{2+}$ injection into the right visual cortex of one normal rat to illustrate the regions of interest for analysis; (B) Comparisons of the normalized signal intensities in the left V1/V2 transition zone among the normal group ( $n=12$ ), left eyelid suturing group (LS; $n=9$ ), and right eyelid suturing group (RS; $n=10$ ); (C) Comparisons of the normalized signal intensities in the left V1/V2 transition zone among the normal group, left eye enucleation group (LE; $\mathbf{n = 1 0}$ ), and right eye enucleation group (RE; $\mathbf{n = 9}$ ). ${ }^{*} p<0.05$, ${ }^{* *} p<0.01, * * * p<0.001$; one-way ANOVA with Bonferroni correction for multiple comparisons; n.s. denotes non-significant difference. Data are shown as mean \pm standard deviation.
The RS group showed a slightly higher, though insignificant, enhancement than the LS group. With the retina removed, only the left eye enucleation (LE) group manifested decreased enhancement in the left V1/V2 transition zone, while the right eye enucleation (RE) group maintained an enhancement level comparable to the normal group (Fig. 4C). These changes were summarized in Fig. 5A. Note that the enhancement decreased more or less equally in all six cortical layers of the left V1/V2 transition zone in the LS, RS, and LE groups (data not shown).

\section{A}
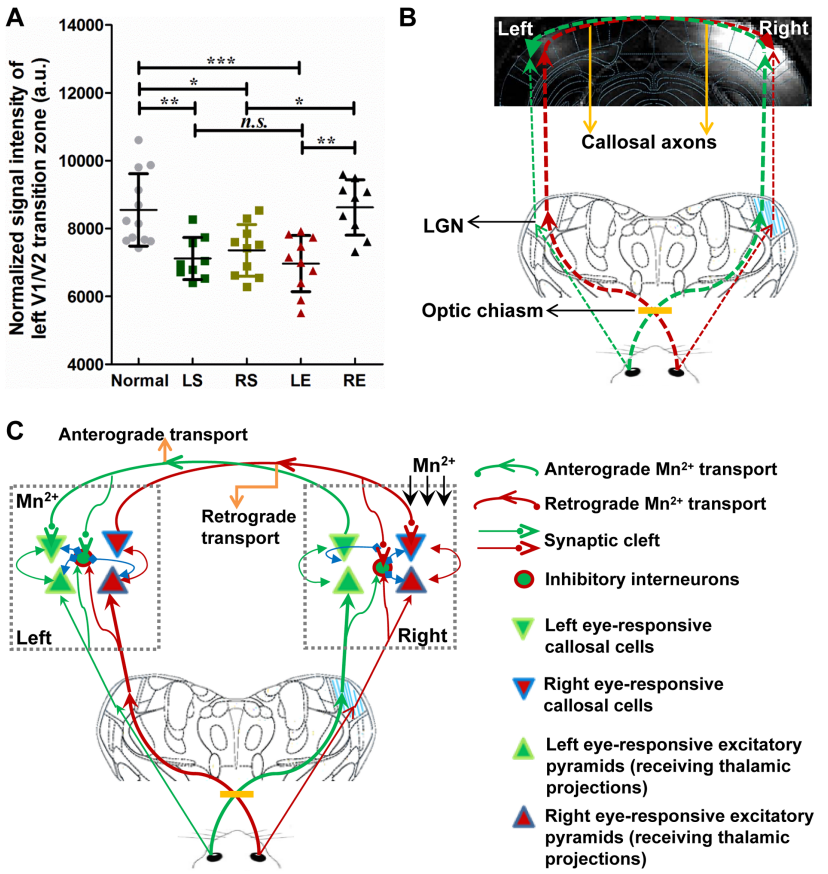

Fig. 5. (A) Summary of the statistical comparisons of the normalized signal intensities in the left V1/V2 transition zone among the normal group $(n=12)$, left eyelid suturing group (LS; $n=9$ ), right eyelid suturing group (RS; $n=10$ ), left eye enucleation group ( $L E, n=10$ ), and right eye enucleation group (RE; $n=9$ ); (B) Illustration of retina projection pathways and transcallosal connections in the bilateral rat brain; (C) Illustration of a simplified visual cortical interhemispheric network and interactions.

\section{DISCUSSION}

Characteristics of interhemispheric $\mathrm{Mn}^{2+}$ transport are highly congruous with previously reported layer-specific transcallosal connections in the visual cortex

This study characterized the layer-specific interhemispheric $\mathrm{Mn}^{2+}$ transport via corpus callosum in the albino rodent brain in vivo. After $\mathrm{Mn}^{2+}$ ions were administered into the right visual cortex, they were transcallosally transported to the left hemisphere, where they collectively accumulated in the V1/V2 transition zone. The most prominent enhancement was observed in layers II/III and V. Temporally, such enhancement symmetrically extended mediallaterally from the V1/V2 border and was confined within approximately $1 \mathrm{~mm}$. These results are in excellent agreement with the past electrophysiological and histological tracing studies $(7,12,18,31$, $44,45)$, clearly indicating that MEMRI can serve as an efficient tool for tracing anatomical interhemispheric connections in vivo.

\section{Mechanisms of interhemispheric $\mathrm{Mn}^{2+}$ transport: axonal transport and synaptic transmission}

Axonal transport of $\mathrm{Mn}^{2+}$ can be anterograde $(21,46)$, retrograde (47), or mixed (34). The callosal cells in the two hemispheres 
could be reciprocally connected by sharing the interhemispheric axonal connections, or interlinked but send independent axonal projections to the contralateral hemisphere (48). The latter scenario is more likely the case as supported by previous histological and electrophysiological findings $(7,19)$. In normal rodents, more than $90 \%$ of the retinal inputs from one eye cross the optic chiasm and project to its contralateral/dominant hemisphere (49) (Fig. 5B), where the visually evoked responses can be anterogradely transported to the other hemisphere (ipsilateral to the eye) via interhemispheric callosal connections $(8,50)$. Therefore, after injection into the right visual cortex, $\mathrm{Mn}^{2+}$ ions can be transported to the left V1/V2 transition zone via anterograde axonal transport through the callosal connections responsible for left eye responses, or via retrograde axonal transport by the callosal connections responsible for right eye responses. For discussion, a simplified cortical interhemispheric connection network in the visual cortex is illustrated in Fig. 5C based on published experimental data ( 8 , 9, 18, 19). After $\mathrm{Mn}^{2+}$ ions are administered into the right visual cortex, they are first uptaken by the local callosal cells, excitatory pyramids, and inhibitory interneurons. Surplus $\mathrm{Mn}^{2+}$ in the callosal cells responsible for left eye responses will be sent directly via anterograde axonal transport to the left hemisphere. Meanwhile, surplus $\mathrm{Mn}^{2+}$ in the excitatory pyramids and interneurons responsible for right eye responses, receiving direct axonal projections from callosal cells in the left hemisphere $(18,19)$, will be first released to the synaptic clefts, and then uptaken by the axonal terminals (synaptic transmission) and retrogradely transported to the originating callosal cells in the left hemisphere. Upon arrival in the left hemisphere, $\mathrm{Mn}^{2+}$ can be further dissipated within the local neuronal network via synaptic transmission.

Previous experiments showed no significant velocity difference between anterograde transport and retrograde transport within the same axons $(51,52) . \mathrm{Mn}^{2+}$ can be transported from the retina to the superior colliculus at a rate of $2.8 \mathrm{~mm} \mathrm{~h}^{-1}$, which includes both the uptake by the retinal ganglion cells and subsequent transport along the optic nerve and tract (53). The interhemispheric distance traced in this study was around $11 \mathrm{~mm}$, which was between the injection site and the left V1/V2 transition zone (42), leading to an estimated transport time of 4 hours. Thus both the 8-hr and 24-hr enhancements can reflect the integration of neuronal $\mathrm{Mn}^{2+}$ uptake in the injected/right hemisphere, transcallosal transport and activitydriven accumulation in the left hemisphere over time (33). The retrograde transport first involves a synaptic transmission of $\mathrm{Mn}^{2+}$ ions before they are packaged into the callosal axons, which may delay their arrival in the left hemisphere. Thus, the 8$\mathrm{hr}$ enhancement may be less influenced by retrograde transport than the 24-hr enhancement. Conceptually, there exists the possibility to distinguish anterograde and retrograde interhemispheric $\mathrm{Mn}^{2+}$ transport by performing dynamic MEMRI early after $\mathrm{Mn}^{2+}$ administration.

\section{Interhemispheric $\mathrm{Mn}^{2+}$ transport is sensitive to activity modulation}

In this study, monocular eyelid suturing resulted in significantly reduced transcallosal $\mathrm{Mn}^{2+}$ labeling of the left visual cortex contralateral to the injection side in adult rats. This finding indicated that interhemispheric $\mathrm{Mn}^{2+}$ transport is sensitive to activity modulation, thus has the potential to serve as a functional biomarker for assessing interhemispheric interactions in vivo. In adult rodents, ocular dominance plasticity is mainly induced in the visual cortex contralateral to the deprived eye, where the deprived eye responses significantly decrease while the open eye responses slightly increase (54). Unlike in juvenile rodents, the visual cortex ipsilateral to the deprived eye is largely unaffected in adult rats (16). Therefore after left eyelid suturing (LS) in the present study, significant decrease of $\mathrm{Mn}^{2+}$ uptake could occur in the injected/right visual cortex, i.e., in the left eye responsive callosal cells that directly contributed to the anterograde axonal $\mathrm{Mn}^{2+}$ transport and in the inhibitory interneurons that directly contributed to the retrograde axonal $\mathrm{Mn}^{2+}$ transport. Meanwhile, slight increase of $\mathrm{Mn}^{2+}$ uptake could arise in the right eye responsive callosal cells which also contributed to the retrograde $\mathrm{Mn}^{2+}$ transport (Fig. 5C). Consequently, the anterograde $\mathrm{Mn}^{2+}$ transport would significantly decrease, while the retrograde $\mathrm{Mn}^{2+}$ transport might not change significantly due to the concurrent $\mathrm{Mn}^{2+}$ uptake decrease by inhibitory interneurons and $\mathrm{Mn}^{2+}$ uptake increase by right eye responsive callosal cells, leading to a net decrease of total $\mathrm{Mn}^{2+}$ transport. On the other hand, right eyelid suturing (RS) would induce limited changes in the injected/right visual cortex. Instead, it could result in significant decrease in right eye responses and slight increase in left eye responses in the left visual cortex. Under these circumstances, $\mathrm{Mn}^{2+}$ uptake in the injected/right hemisphere was not affected significantly, but $\mathrm{Mn}^{2+}$ accumulation decreased significantly in the left visual cortex, i.e., in right eye responsive neurons that directly received the retrogradely transported $\mathrm{Mn}^{2+}$, accompanied by slight $\mathrm{Mn}^{2+}$ accumulation increase in left eye responsive neurons that directly received the anterogradely transported $\mathrm{Mn}^{2+}$ (Fig. 5C), leading to a net decrease of the total $\mathrm{Mn}^{2+}$ accumulation. As discussed above, retrograde $\mathrm{Mn}^{2+}$ transport first involves a transynaptic transmission of $\mathrm{Mn}^{2+}$ ions in the right hemisphere. Thus, it is less efficient than anterograde transport and is likely to contribute less to the overall transcallosal $\mathrm{Mn}^{2+}$ labeling. This may explain why transcallosal $\mathrm{Mn}^{2+}$ labeling was less reduced by RS than by LS (though insignificant) in the present study. Taken together, the decreased transcallosal $\mathrm{Mn}^{2+}$ labeling of the left V1/V2 transition zone in the LS group was largely caused by the decreased anterograde axonal transport due to the neuronal activity changes in the right hemisphere. In the RS group, it was likely caused by the decreased accumulation of the retrogradely transported $\mathrm{Mn}^{2+}$ due to the neuronal activity changes in the left hemisphere.

\section{Interhemispheric $\mathrm{Mn}^{2+}$ transport is modulated differently upon eyelid suturing and monocular eye enucleation}

Transcallosal $\mathrm{Mn}^{2+}$ labeling of the left V1/V2 transition zone after intracortical $\mathrm{Mn}^{2+}$ injection into the right visual cortex was significantly reduced by both LS and RS, and by left eye enucleation (LE) but not by right eye enucleation (RE), supporting that the rat brain may recruit different mechanisms for its adaptations to monocular eyelid suturing and enucleation. Similar to monocular eyelid suturing, monocular enucleation could result in significant decrease of the visual evoked responses in the contralateral visual cortex (55). Therefore, similar to LS and RS, left eye enucleation (LE) could result in significant anterograde $\mathrm{Mn}^{2+}$ transport decrease while right eye enucleation (RE) could lead to significant $\mathrm{Mn}^{2+}$ uptake decrease in right eye responsive neurons in the left V1/V2 transition zone. In contrast to monocular eyelid suturing, monocular enucleation could cause more rapid structural changes of the inhibitory neurons including dendritic branch retractions, resulting in stronger disinhibition of the cortical circuit $(54,56)$, thus more significant enhancement of the open eye responses in the hemisphere contralateral to the enucleated eye (13). 
Therefore in this study, left eye enucleation (LE) could induce stronger enhancement of the right eye responses in the injected/right hemisphere than LS. However, only the callosal cells would directly contribute to the retrograde $\mathrm{Mn}^{2+}$ transport (Fig. 5C). Due to the concurrent strong depression of inhibitory neuronal responses, the retrograde $\mathrm{Mn}^{2+}$ transport may not increase significantly to offset the decrease of anterograde transport (Fig. 5C), resulting in a net decrease of transcallosal $\mathrm{Mn}^{2+}$ labeling. On the other hand, right eye enucleation (RE) could cause more significant enhancement of the left eye responses in the left hemisphere than RS. The increased left eye responses, in both callosal cells and excitatory pyramids receiving thalamic projections, could contribute to significant increase of $\mathrm{Mn}^{2+}$ accumulation that offsetted the accumulation decrease caused by right eye response depression. As a result, the transcallosal $\mathrm{Mn}^{2+}$ labeling was maintained in the normal range. Taken together, these findings suggested that interhemispheric $\mathrm{Mn}^{2+}$ transport is predominately modulated by the anterograde transcallosal axonal transport and activity changes in the hemisphere contralateral to the injection side.

The transcallosal $\mathrm{Mn}^{2+}$ labeling of the left V1/V2 transition zone decreased by more or less the same extent in all six cortical layers in the LS, RS, and LE groups. This finding is consistent with a previous report that monocular deprivation in adult mice could induce ocular dominance modifications simultaneously in different cortical layers (15). Nevertheless, this could be partly related to the spatial resolution, specific imaging time points, and high $\mathrm{Mn}^{2+}$ concentration employed in the current study. To investigate the potential layer-specific changes under different experiential or chemical manipulations, future MEMRI studies may be performed by employing more advanced $\mathrm{Mn}^{2+}$ administration methods, e.g., micro-infusion using osmotic pumps (57).

\section{Conclusion}

This study for the first time characterized the layer-specific interhemispheric connections of the visual cortex in living rodent brains using MEMRI. The results demonstrated MEMRI as an efficient tool for investigating interhemispheric interactions both anatomically and functionally. Although the translation of our findings on murine models into humans warrants further investigation, we speculate by analogy that the adult brain may recruit differential adaptive mechanisms following eyelid suturing and enucleation. Future research will further enhance our understanding of transcallosal interhemispheric excitation and inhibition, and provide promising directions for clinical rehabilitation for conditions such as amblyopia and functional visuospatial neglect resulted from unilateral brain damage.

\section{MATERIALS AND METHODS}

\section{Animals}

Seven- to eight-week-old normal male Sprague Dawley rats (Charles River Lab, USA) were obtained from the institutional laboratory animal unit and randomly divided into five groups: normal control group $(\mathrm{n}=12)$, left eyelid suturing group (LS; $\mathrm{n}=9$ ), right eyelid suturing group (RS; $\mathrm{n}=10)$, left eye enucleation group (LE; $\mathrm{n}=10$ ), and right eye enucleation group (RE; $\mathrm{n}=9$ ). Monocular eyelid suturing with intact retina or eye enucleation $(8,55)$ was performed seven days before $\mathrm{Mn}^{2+}$ injection. The LS and RS groups were checked daily to ensure that the sutured eyelids were not open. Buprenorphine $(0.1 \mathrm{mg} / \mathrm{kg})$ was given subcutaneously as analgesic for 3 days after the surgery. The sutured eyelids were maintained shut until the end of the experiment. All rats were raised under a 12:12 hour light-dark cycle with ad libitum access to standard food and water. All the experimental procedures were approved by the local institutional animal ethics committee.

\section{$\mathrm{MnCl}_{2}$ administration and MRI}

$\mathrm{MnCl}_{2}$ (500 mM, $\left.100 \mathrm{nl}, \mathrm{pH}=7.4\right)$ (Sigma-Aldrich, USA) was stereotaxically injected over $10 \mathrm{~min}$ into the right V1/V2 border of each rat at $6.5 \mathrm{~mm}$ posterior and $5.5 \mathrm{~mm}$ lateral to bregma with a 1.0 $\mu \mathrm{L}$ Hamilton syringe under inhaled isoflurane anesthesia (4\% for induction and $2 \%$ for maintenance) (41). $\mathrm{MnCl}_{2}$ dosage of $500 \mathrm{mM}$ $100 \mathrm{nl}$ was selected for achieving quality labeling with minimized toxicity based on preliminary experiments (data not shown). The syringe was kept in place for 5 min after injection and then gently removed. In the normal group, MRI was performed at $24 \mathrm{hrs}$ before, $8 \mathrm{hrs}(\mathrm{n}=5)$, and $24 \mathrm{hrs}(\mathrm{n}=12)$ after $\mathrm{Mn}^{2+}$ injection, with 3 rats also imaged at $48 \mathrm{hrs}$ after injection. In the other groups, MEMRI was performed only at $24 \mathrm{hrs}$ after $\mathrm{Mn}^{2+}$ injection. Time points for MRI imaging were chosen to best capture the dynamics of manganese ion uptake, transport, and clearance as characterized by previous MEMRI studies $(27,58)$.

All MRI experiments were performed on a 7T MRI animal scanner (70/16 PharmaScan, Bruker Biospin GmbH, Germany) using a 72-mm birdcage transmit-only RF coil and an actively decoupled receive-only quadrature surface coil. During MRI, rats were anesthetized with isoflurane (3\% for induction and $1.5 \%$ for maintenance) with respiration monitoring and were kept warm at $37^{\circ} \mathrm{C}$ with circulating water (30). MDEFT images were acquired for characterizing $\mathrm{Mn}^{2+}$ enhancement $(35,59)$ with $\mathrm{TR}=4000 \mathrm{~ms}$, Echo $\mathrm{TR} / \mathrm{TE}=12 / 4 \mathrm{~ms}$, inversion time $(\mathrm{TI})=1100 \mathrm{~ms}$, number of segments $=4$, number of averages $=8$, FOV $=32 \times 32 \mathrm{~mm}^{2}$, matrix $=256 \times 256$, slice thickness $=0.5 \mathrm{~mm}$, no slice gap, number of slices $=28$, and $60 \mathrm{~min}$ acquisition time.

\section{Data processing and analysis}

To depict interhemispheric $\mathrm{Mn}^{2+}$ transport via corpus callosum, MDEFT images from normal animals were normalized by background noise level and reconstructed three-dimensionally using AMIRA 5.2.2 (Visage Imaging Inc.). For semi-quantitative analysis of the contralateral enhancement patterns at both $8 \mathrm{hrs}$ and 24 $\mathrm{hrs}$ in the normal group, regions of interest (ROIs) were manually delineated in five consecutive MDEFT slices containing the most prominent left hemisphere enhancement. ROIs were defined as a stripe along the cortical depth direction over the most enhanced pixels or medial-laterally along the cortical surface direction. Signal intensity profiles of these ROIs were plotted using ImageJ, first averaged among five slices in each animal and then averaged among the animals in each group. To examine the left V1/V2 transition zone enhancement difference between the normal and deprived groups, ROIs were placed over the most prominently enhanced left V1/V2 transition zone in five consecutive MDEFT slices by referencing to a rat brain atlas (59). ROIs were also defined in the right posterior cortex while avoiding the injection site. To minimize contamination by potential variation of injected $\mathrm{Mn}^{2+}$ dosages, signal intensities of the ROIs of the left V1/V2 transition zone were normalized by those of the right posterior cortex. Normalized and mean signal intensities of these ROIs were compared among groups using one-way analysis of variance (ANOVA) with Bonferroni correction for multiple comparisons. Statistical significance was accepted at $p<0.05$. 


\section{REFERENCES}

1. J. Faguet, B. Maranhao, S. L. Smith, J. T. Trachtenberg, Ipsilateral eye cortical maps are uniquely sensitive to binocular plasticity. J. Neurophysiol. 101, 855-861 (2009).

2. A. Sale, J. F. M. Vetencourt, P. Medini, M. C. Cenni, L. Baroncelli, R. de Pasquale, L. Maffei, Environmental enrichment in adulthood promotes amblyopia recovery through a reduction of intracortical inhibition. Nat. Neurosci. 10, 679-681 (2007).

3. J. L. Chen, W. C. Lin, J. W. Cha, P. T. So, Y. Kubota, E. Nedivi, Structural basis for the role of inhibition in facilitating adult brain plasticity. Nat. Neurosci. 14, 587-594 (2011)

4. R. J. Rushmore, A. Valero-Cabre, S. G. Lomber, C. C. Hilgetag, B. R. Payne, Functional circuitry underlying visual neglect. Brain 129, 1803-1821 (2006).

5. J. S. Espinosa, M. P. Stryker, Development and plasticity of the primary visual cortex. Neuron 75, 230-249 (2012)

6. J. Olavarria, R. C. van Sluyters, Widespread callosal connections in infragranular visual cortex of the rat. Brain Res. 279, 233-237 (1983).

7. G. M. Innocenti, D. Aggoun-Zouaoui, P. Lehmann, Cellular aspects of callosal connections and their development. Neuropsychologia 33, 961-987 (1995).

8. L. Restani, C. Cerri, M. Pietrasanta, L. Gianfranceschi, L. Maffei, M. Caleo, Functional masking of deprived eye responses by callosal input during ocular dominance plasticity. Neuron $\mathbf{6 4}$ 707-718 (2009).

9. J. F. Olavarria, Callosal connections correlate preferentially with ipsilateral cortical domain in cat areas 17 and 18, and with contralateral domains in the 17/18 transition zone. J. Comp. Neurol. 433, 441-457 (2001).

10. B. Zhang, H. Bi, E. Sakai, I. Maruko, J. Zheng, E. L. Smith3rd, Y. M. Chino, Rapid plasticity of binocular connections in developing monkey visual cortex (V1). Proc. Natl. Acad. Sci. U.S.A. 102, 9026-9031 (2005)

11. Y. Morishima, M. Toigawa, N. Ohmura, T. Yoneda, Y. Tagane, Y. Hata, Critical period of experience-driven axon retraction in the pharmacologically inhibited visual cortex. Cereb. Cortex 23, 2423-2428 (2013).

12. A. M. Grigonis, E. H. Murphy, Organization of callosal connections in the visual cortex of the rabbit following neonatal enucleation, dark rearing, and strobe rearing. J. Comp. Neurol. 312 561-572 (1991).

13. M. Y. Frenkel, M. F. Bear, How monocular deprivation shifts ocular dominance in visual cortex of young mice. Neuron 44, 917-923 (2004).

14. P. Medini, Layer- and cell-type-specific subthreshold and suprathreshold effects of long-term monocular deprivation in rat visual cortex. J. Neurosci. 31, 17134-17148 (2011).

15. C. H. Liu, A. J. Heynen, M. G. Hussain-Shuler, M. F. Bear, Cannabinoid receptor blockade reveals parallel plasticity mechanisms in different layers of mouse visual cortex. Neuron $\mathbf{5 8}, 340-345$ (2008).

16. M. Sato, M. P. Stryker, Distinctive features of adult ocular dominance plasticity. J. Neurosci. 28, 10278-10286 (2008)

17. C. D. Rittenhouse, H. Z. Shouval, M. A. Paradiso, M. F. Bear, Monocular deprivation induce homosynaptic long-term depression in visual cortex. Nature 397, 347-350 (1999).

18. L. Petreanu, D. Huber, A. Sobczyk, K. Svoboda, Channelrhodopsin-2-assisted circuit mappin of long-range callosal projections. Nat. Neurosci. 10, 663-668 (2007).

19. T. Karayannis, I. Huerta-Ocampo, M. Capogna, GABAergic and pyramidal neurons of deep cortical layers directly receive and differently integrate callosal input. Cereb. Cortex 17, 12131226 (2007).

20. C. A. Massaad, R. G. Pautler, Manganese-enhanced magnetic resonance imaging (MEMRI). Methods Mol. Biol. 711, 145-174 (2011).

21. R. G. Pautler, A. C. Silva, A. P. Koretsky, In vivo neuronal tract tracing using manganeseenhanced magnetic resonance imaging. Magn. Reson. Med. 40, 740-748 (1998).

22. R. G. Pautler, A. P. Koretsky, Tracing odor-induced activation in the olfactory bulbs of mice using manganese-enhanced magnetic resonance imaging. Neurolmage 16, 441-448 (2002)

23. B. H. de la Garza, G. Li, Y.-Y. I. Shih, T. Q. Duong, Layer-specific manganese-enhanced MRI of the retina in light and dark adaptation. Invest. Opthalmol. Vis. Sci. 53, 4352-4358 (2012).

24. T. Q. Duong, A. C. Silva, S. P. Lee, S. G. Kim, Functional MRI of calcium-dependent synaptic activity: Cross correlation with CBF and BOLD measurements. Magn. Reson. Med. 43, 383392 (2000).

25. X. Yu, J. Zou, J. S. Babb, G. Johnson, D. H. Sanes, D. H. Turnbull, Statistical mapping of sound evoked activity in the mouse auditory midbrain using Mn-enhanced MRI. Neurolmage 39, 223-230 (2008).

26. D. Bissig, B. A. Berkowitz, Same-session functional assessment of rat retina and brain with manganese-enhanced MRI. Neurolmage 58, 749-760 (2011).

27. X. Yu, Y. Z. Wadghiri, D. H. Sanes, D. H. Turnbull, In vivo auditory brain mapping in mice with Mn-enhanced MRI. Nat. Neurosci. 8, 961-968 (2005).

28. A. van der Linden, V. van Meir, I. Tindemans, M. Verhoye, J. Balthazart, Applications of manganese-enhanced magnetic resonance imaging (MEMRI) to image brain plasticity in song birds. NMR Biomed. 17, 602-612 (2004).

29. X. Yu, S. Chung, D. Y. Chen, S. Wang, S. J. Dodd, J. R. Walters, J. T. Isaac, A. P. Koretsky, Thalamocortical inputs show post-critical-period plasticity. Neuron 74, 731-742 (2012).

30. J. Yang, E. X. Wu, Detection of cortical gray matter lesion in the late phase of mild hypoxicischemic injury by manganese-enhanced MRI. Neurolmage 39, 669-679 (2008).

31. T. Inoue, T. Majid, R. G. Pautler, Manganese enhanced MRI (MEMRI): Neurophysiological applications. Rev. Neurosci. 22, 675-694 (2011).

32. K. C. Chan, J. Li, P. Kau, I. Y. Zhou, M. M. Cheung, C. Lau, J. Yang, K. F. So, E. X. Wu, In vivo retinotopic mapping of superior colliculus using manganese-enhanced magnetic resonance imaging. Neurolmage 54, 389-395 (2011).

33. T. Watanabe, J. Frahm, T. Michaelis, Manganese-enhanced MRI of the mouse auditory pathway. Magn. Reson. Med. 60, 210-212 (2008).
34. R. G. Pautler, R. Mongeau, R. E. Jacobs, In vivo trans-synaptic tract tracing from the murine striatum and amygdala utilizing manganese enhanced MRI (MEMRI). Magn. Reson. Med. 50 33-39 (2003).

35. J. Tucciarone, K.-H. Chuang, S. J. Dodd, A. Silva, G. Pelled, A. P. Koretsky, Layer specific tracing of corticocortical and thalamocortical connectivity in the rodent using manganese enhanced MRI. Neurolmage 44, 923-931 (2009).

36. D. Bissig, B. A. Berkowitz, Manganese-enhanced MRI of layer-specific activity in the visual cortex from awake and free-moving rats. Neurolmage 44, 627-635 (2009).

37. A. van der Linden, M. Verhoye, V. van Meir, I. Tindemans, M. Eens, P. Absil, J. Balthazart, In vivo manganese-enhanced magnetic resonance imaging reveals connections and functional properties of the songbird vocal control system. Neuroscience 112, 467-474 (2002).

38. M. Bilgen, W. Peng, B. Al-Hafez, N. Dancause, Y.-Y. He, P. D. Cheney, Electrical stimulation of cortex improves corticospinal tract tracing in rat spinal cord using manganese-enhanced MRI. J. Neurosci. Methods 156, 17-22 (2006).

39. E. L. Bearer, T. L. Falzone, X. Zhang, O. Biris, A. Rasin, R. E. Jacobs, Role of neuronal activity and kinesin on tract tracing by manganese-enhanced MRI (MEMRI). Neurolmage 37, S37-S46 (2007).

40. A. S. Lowe, I. D. Thompson, N. R. Sibson, Quantitative manganese tract tracing: Dosedependent and activity-independent terminal labelling in the mouse visual system. NMR Biomed. 21, 859-867 (2008).

41. K. C. Chan, J. S. Cheng, S. Fan, I. Y. Zhou, J. Yang, E. X. Wu, In vivo evaluation of retinal and callosal projections in early postnatal development and plasticity using manganese-enhanced MRI and diffusion tensor imaging. Neurolmage 59, 2274-2283 (2012).

42. G. Paxinos, C. Watson, The Rat Brain in Stereotaxic Coordinates (Elsevier Academic Press, 2005).

43. S. Failor, V. Nguyen, D. P. Darcy, J. Cang, M. F. Wendland, M. P. Stryker, P. S. McQuillen, Neonata cerebral hypoxia-ischemia impairs plasticity in rat visual cortex. J. Neurosci. 30, 81-92 (2010)

44. H. Mizuno, T. Hirano, Y. Tagawa, Evidence for activity-dependent cortical wiring: Formation of interhemispheric connections in neonatal mouse visual cortex requires projection neuron activity. J. Neurosci. 27, 6760-6770 (2007).

45. B. Dreher, C. Dehay, J. Bullier, Bihemispheric collateralization of the cortical and subcortical afferents to the rat's visual cortex. Eur. J. Neurosci. 2, 317-331 (1990).

46. M. Bilgen, Imaging corticospinal tract connectivity in injured rat spinal cord using manganeseenhanced MRI. BMC Med. Imaging 6, 15 (2006).

47. K. Matsuda, H. X. Wang, C. Suo, D. McCombe, M. K. Horne, W. A. Morrison, G. F. Egan, Retrograde axonal tracing using manganese enhanced magnetic resonance imaging. Neurolmage 50, 366-374 (2010).

48. G. M. Innocenti, Sensory-Motor Areas and Aspects of Cortical Connectivity, E. G. Jones, A. Peters, eds. (Springer US, 1986), pp. 291-353.

49. C. Cerri, L. Restani, M. Caleo, Callosal contribution to ocular dominance in rat primary visual cortex. Eur. J. Neurosci. 32, 1163-1169 (2010).

50. J. W. Lewis, J. F. Olavarria, Two rules for callosal connectivity in striate cortex of the rat. J. Comp. Neurol. 361, 119-137 (1995).

51. K. Buchner, D. Seitz-Tutter, K. Schönitzer, D. G. Weiss, A quantitative study of anterograde and retrograde axonal transport of exogenous proteins in olfactory nerve C-fibers. Neuroscience 22, 697-707 (1987)

52. S. T. Brady, K. K. Pfister, G. S. Bloom, A monoclonal antibody against kinesin inhibits both anterograde and retrograde fast axonal transport in squid axoplasm. Proc. Natl. Acad. Sci. U.S.A. 87, 1061-1065 (1990).

53. T. Watanabe, J. Frahm, T. Michaelis, Functional mapping of neural pathways in rodent brain in vivo using manganese-enhanced three-dimensional magnetic resonance imaging. NMR Biomed. 17, 554-568 (2004).

54. K. Kameyama, K. Sohya, T. Ebina, A. Fukuda, Y. Yanagawa, T. Tsumoto, Difference in binocularity and ocular dominance plasticity between GABAergic and excitatory cortical neurons. J. Neurosci. 30, 1551-1559 (2010).

55. L. van Brussel, A. Gerits, L. Arckens, Evidence for cross-modal plasticity in adult mouse visual cortex following monocular enucleation. Cereb. Cortex 21, 2133-2146 (2011).

56. T. Keck, V. Scheuss, R. I. Jacobsen, C. J. Wierenga, U. T. Eysel, T. Bonhoeffer, M. Hübener, Loss of sensory input causes rapid structural changes of inhibitory neurons in adult mouse visual cortex. Neuron 71, 869-882 (2011).

57. S. Canals, M. Beyerlein, A. L. Keller, Y. Murayama, N. K. Logothetis, Magnetic resonance imaging of cortical connectivity in vivo. Neurolmage 40, 458-472 (2008).

58. K. C. Chan, S. J. Fan, R. W. Chan, J. S. Cheng, I. Y. Zhou, E. X. Wu, In vivo visuotopic brain mapping with manganese-enhanced MRI and resting-state functional connectivity MRI. Neurolmage 90, 235-245 (2014)

59. R. Deichmann, Optimized RF excitation for anatomical brain imaging of the occipital lobe using the 3D MDEFT sequence and a surface transmit coil. Magn. Reson. Med. 53, 1212-1216 (2005).

Acknowledgments: Funding: This work was supported by Hong Kong Research Grant Council, Lam Woo Foundation, Guangdong Key Technologies for Treatment of Brain Disorders (2018B030332001 to E.X. Wu), Guangdong-Hong Kong-Macao Greater Bay Area Center for Brain Science and Brain-Inspired Intelligence Fund (2019008 to A.T.L. Leong), and HKU Seed Fund for Basic Research (104005866 to A.T.L. Leong). Author contributions: S.F. and E.X.W. designed the study. S.F. performed the experiments. S.F. and E.X.W. analyzed the data. S.F., A.T.L.L., and E.X.W. interpreted the data. S.F., A.T.L.L., and E.X.W. wrote the manuscript. E.X.W. supervised the study Competing interests: The authors declare that they have no competing interests. Data and materials availability: All data and materials are available upon request to the corresponding author. 